Zhixin Xiong

Peilin Luo

Xiaochuan $Y u$

https://doi.org/10.21278/TOF.43102

ISSN 1333-1124

eISSN 1849-1391

\title{
A STUDY ON THE EXPLICIT EXPRESSION OF CRITICAL STRESS AND EULER STRESS AND ITS APPLICATION
}

\begin{abstract}
Summary
Both the tangent modulus theory and the double modulus theory are classical theories which can be applied to the elastic-plastic stability analysis of columns. In the traditional tangent modulus theory, numerous iterations are required to calculate the critical buckling stress and this makes the method very time-consuming. In this paper, an explicit formula for establishing a direct correlation between the critical stress and the Euler stress has been proposed to reduce trial calculations. This formula can be applied to spherical shells by simplifying their stiffened plates to the form of beams on elastic foundations. The explicit expressions of both modulus theories can be used to calculate the ultimate strength of a spherical shell under pressure. The results from the proposed expression are compared with experimental results and other numerical results.
\end{abstract}

Key words: $\quad$ tangent modulus; double modulus; beam on an elastic foundation; spherical shell; critical stress

\section{Introduction}

The Euler formula, which was formed to study the elastic stability of a compression bar [1], has been widely applied to various types of structures under pressure. For a long time, it had been believed that that formula was not only applicable to a slender bar but also to a short column. In the nineteenth century, some experiments showed that it was dangerous to apply the Euler formula to a short column; additionally, it was shown that that formula could be applied in the elastic stage. When buckling or critical stress is beyond the proportional limit, the material nonlinearity should be considered. This phenomenon is named elasto-plastic buckling. Engesser presented the tangent modulus theory which suggested varying the deformation modulus to replace the elastic modulus of the Euler formula [2]. There were different stress-strain relationships because compressive stress increases in concave and reduces in convex structures when the structure is slightly bending. To tackle this problem, Considère extended the concept of double modulus in 1891. Later, Engesser proposed the double modulus theory and suggested the use of the converted modulus to calculate buckling loads [3]. This converted modulus is related to the tangent modulus and the double modulus. 
In 1910, von Karman [4] modified the Considère-Engesser theory and applied it to the evaluation of the double modulus for rectangular and idealized H-sections. The results were compared with experimental tests. Those experimental data were more consistent with the value from the tangent modulus theory. Shanley presented his model in 1946[5]. Based on that mechanical model, Shanley established the relationship between loads after buckling and deflection [6]. Through that, it was proved that two kinds of ultimate loads existed. One is called the tangent modulus load and the other is the double modulus load. The former is the lower limit value and the latter is the upper limit value [7, 8]. The tangent modulus is applied to calculate the buckling strength. In the 1950s, Duberg and Wilder showed that for common engineering materials the maximum load carrying capability of the column is only slightly above the tangent modulus bifurcation point [9]. In 1972, Hutchinson analysed axisymmetric plastic buckling in a spherical shell under pressure; the shell had various axisymmetric imperfections. As the imperfection amplitude approaches zero, the collapse load rises very slightly above the tangent modulus buckling load [10]. Reynolds proposed an approximate formula based on Gerard's differential equations for the plastic buckling of cylindrical shells to be used for buckling in the inelastic region [11]. According to that formula, the buckling pressure is a function of the cylinder geometry and the secant and tangent moduli as determined from the stress-strain intensity diagram for the shell material. Later, Krenzke and Kiernan completed four series of tests for more than 200 small spherical models with different imperfections; they derived the empirical formula with the tangent and the secant modulus [12]. Schneider et al. proposed a procedure and formulae for calculating the tangent modulus from the actual stress-strain curve [13]. Pranesh et al. used the tangent modulus theory to calculate the inelastic buckling strength of a spherical shell under pressure; their results are close to those of the theoretical and the numerical method [14]. Luo established the equilibrium equation of a spherical cap subjected to hydrostatic pressure; that equation is the same as that for a beam on an elastic foundation subjected to axial and lateral loads [15]. Generally speaking, Luo tried to expand Hooke's law and propose a more generalized theory based on the tangent modulus [16]. When using the tangent modulus to calculate the critical stress, some trials were needed to get a suitable tangent modulus.

In order to reduce this tedious process, an explicit expression for the tangent modulus or the double modulus has been explicitly introduced in this paper. The relationship between the critical stress and the Euler stress can be established directly. The explicit expressions of both modulus theories can be used to calculate the ultimate strength of a spherical shell under pressure.

\section{The tangent modulus and the double modulus theory}

\subsection{The tangent modulus theory}

The relationship between the actual critical stress $\sigma_{c r}$ and the Euler stress $\sigma_{E}$ of structures under pressure can be determined by several modulus theories; one of them is the tangent modulus theory whose results can be consistent with experimental results. The tangent modulus theory can be expressed as follows:

$$
\sigma_{c r}=E_{t} \sigma_{E} / E
$$

where $\quad E_{t}=\left.\frac{d \sigma}{d \varepsilon}\right|_{\sigma=\sigma_{c r}}$ 


\subsection{The double modulus theory}

Another modulus theory is the double modulus theory, which is sometimes called the reduced modulus theory. In the double modulus theory, the relationship between the Euler stress and the critical stress can be expressed as:

$$
\sigma_{c r}=E_{r} \sigma_{E} / E
$$

where $\quad E_{r}=\frac{4 E E_{t}}{\left(\sqrt{E}+\sqrt{E_{t}}\right)^{2}}$ is the rectangular cross section.

From Eq. (1) and (3), the tangent modulus $E_{t}$ should be calculated first in order to get the critical stress $\sigma_{c r}$. $E_{t}$ is correlated with the critical stress by the stress-strain curve.

\section{The explicit expression for the critical stress and the Euler stress}

\subsection{A formula for the stress-strain curve}

In order to use the modulus theory, it is necessary to establish a formula for the stressstrain curve, such as the Ramberg-Osgood relational expression [17, 18], three parameter solution $\left(E, \sigma_{0.7}, \sigma_{0.8}\right)[19]$, and so on. In this paper, a power function is used to describe the stress-strain curve

$$
\sigma=\sigma_{0}\left[A-B\left(\frac{\sigma_{0}}{E \varepsilon}\right)^{-c}\right]^{m},
$$

where $A, B, c$, and $m$ are four constants which can represent the nonlinear properties of the material. The values of $A, B, c$, and $m$ are obtained from eigenvalue analysis using the method of least squares. In the linear region, they are: $A=0, B=-1, c=m=1$.

Eq. (5) can be also written as:

$$
\bar{\sigma}=\left[A-B(\bar{\varepsilon})^{c}\right]^{m}
$$

where $\bar{\sigma}=\sigma / \sigma_{0}(7)$

$$
\bar{\varepsilon}=E \varepsilon / \sigma_{0}
$$

$\bar{\sigma}$ and $\bar{\varepsilon}$ are dimensionless parameters.

The following formula can be used to describe the stress of limit equilibrium state.

$$
\bar{\sigma}_{c r}=\left[A-B\left(\bar{\varepsilon}_{c r}\right)^{c}\right]^{m}
$$

where $\quad \bar{\sigma}_{c r}=\sigma_{c r} / \sigma_{0}$

$$
\bar{\varepsilon}_{c r}=E \varepsilon_{c r} / \sigma_{0}
$$


From the stress-strain curve, the dimensionless tangent modulus $E_{t}$ can be derived as follows:

$$
\frac{\mathrm{d} \bar{\sigma}}{\mathrm{d} \bar{\varepsilon}}=\frac{\mathrm{d}\left(\sigma / \sigma_{0}\right)}{\mathrm{d}\left(E \varepsilon / \sigma_{0}\right)}=\frac{1}{E} \frac{\mathrm{d} \sigma}{\mathrm{d} \varepsilon}=\frac{E_{t}}{E}=\bar{E}_{t}
$$

Therefore, the relationship between $\sigma_{c r}$ and $E_{t}$ can be expressed as:

$$
\frac{\bar{\sigma}_{c r}}{\bar{\sigma}_{E}}=\frac{\mathrm{d} \bar{\sigma}}{\mathrm{d} \bar{\varepsilon}}
$$

where $\quad \bar{\sigma}_{E}=\sigma_{E} / \sigma_{0}$

Figure 1 shows two types of dimensionless stress-strain curves, where the curve $\bar{\sigma}_{L}\left(\bar{\varepsilon}_{L}\right)$ can be applied to an ideal elastic body and the curve $\bar{\sigma}(\bar{\varepsilon})$ represents the actual stress-strain relationship which considers the nonlinearity of the material.

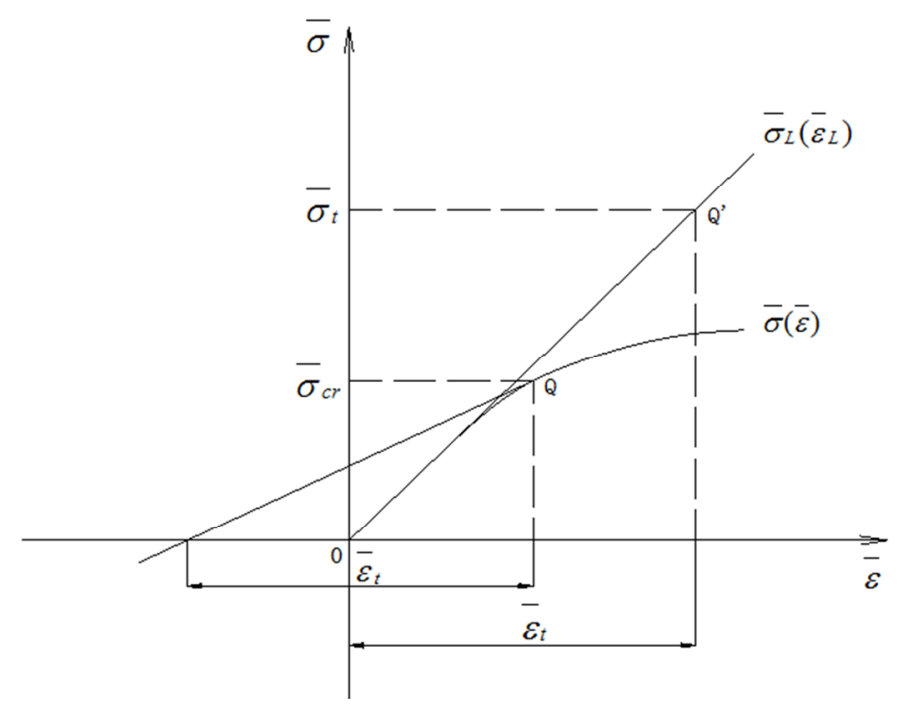

Fig. 1 The stress-strain $(\bar{\sigma} \sim \bar{\varepsilon})$ curve and the tangent modulus strain $\left(\bar{\varepsilon}_{t}\right)$

For the curve $\bar{\sigma}_{L}\left(\bar{\varepsilon}_{L}\right)$

$$
\bar{\varepsilon}_{t}=\bar{\sigma}_{t}
$$

where $\quad \bar{\sigma}_{L}=\sigma_{L} / \sigma_{0}$

$$
\begin{aligned}
& \bar{\varepsilon}_{L}=E \varepsilon_{L} / \sigma_{0} \\
& \bar{\varepsilon}_{t}=E \varepsilon_{t} / \sigma_{0} \\
& \bar{\sigma}_{t}=\sigma_{t} / \sigma_{0}
\end{aligned}
$$

For the curve $\bar{\sigma}(\bar{\varepsilon})$

$$
\bar{\sigma}=f(\bar{\varepsilon})
$$


If the point $\mathrm{Q}$ is the limit point, the tangential line may be mathematically expressed as:

$$
\left.\frac{\mathrm{d} \bar{\sigma}}{\mathrm{d} \bar{\varepsilon}}\right|_{\bar{\sigma}=\bar{\sigma}_{c r}}=\frac{\bar{\sigma}_{c r}}{\bar{\varepsilon}_{t}}
$$

It can be easily re-written as:

$$
\bar{\sigma}_{c r}=f\left(\bar{\varepsilon}_{t}\right)
$$

3.2 The tangent modulus

As shown in Fig.2, it is easy to derive the formula for the tangent strain.

$$
\begin{aligned}
& \varepsilon_{t}=\frac{\sigma}{E_{t}}=\frac{\sigma \mathrm{d} \varepsilon}{\mathrm{d} \sigma}=\frac{\mathrm{d} W}{\mathrm{~d} \sigma}=W_{\sigma}^{\prime} \\
& \bar{\varepsilon}_{t}=\frac{\varepsilon_{t}}{\varepsilon_{0}}=\frac{1}{\varepsilon_{0}} W_{\sigma}^{\prime}
\end{aligned}
$$

where $\mathrm{d} W=\sigma \mathrm{d} \varepsilon$ is the increment in the value of strain energy density $W$.

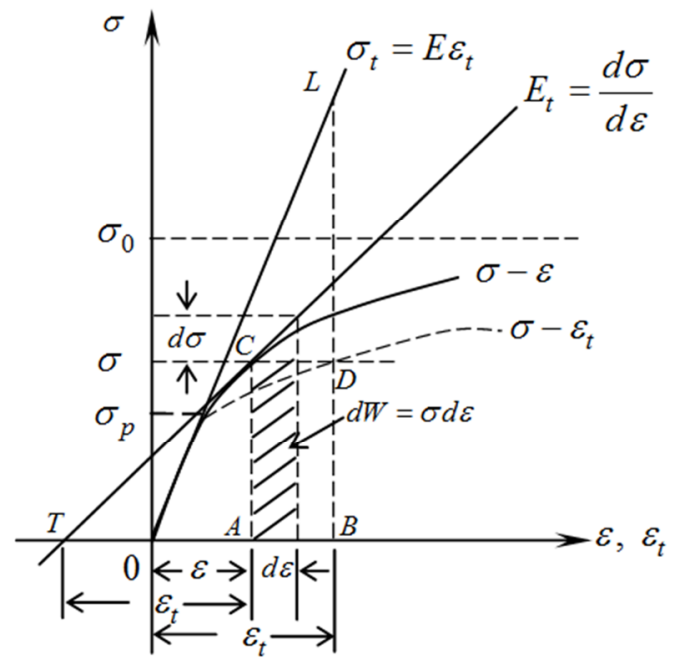

Fig. 2 The stress-strain $(\sigma \sim \varepsilon)$ curve and the $\sigma \sim \varepsilon_{t}$ curve

Energy density is an important characteristic parameter of equilibrium states. From Eq. (24), the parameter $\varepsilon_{t}$ is the derivative of strain energy density, which corresponds to the strain energy density. It is also shown that $\bar{\varepsilon}_{t}$ is another parameter characterizing the status of equilibrium. Namely, the essence of the tangent modulus theory is the equality of tangent strain among different conditions, including the actual condition with the nonlinear or perfect elastic state; the tangent strain $\bar{\varepsilon}_{t}$ can be considered as a standard of the structure buckling failure.

\subsection{A newly-proposed expression for the critical stress and the Euler stress}

From Fig.1, one can see that the point Q' corresponds to a special case in which the Euler load is also the critical load of the structure. Therefore, the strain $\bar{\varepsilon}_{t}$ will be equal to $\bar{\sigma}_{t}$.

From the stress-strain curve $\bar{\sigma}_{L}\left(\bar{\varepsilon}_{L}\right)$ in Fig. 1, we have

$$
\bar{\varepsilon}_{t}=\bar{\sigma}_{E}
$$


From Eq. (25) and (22), one can derive

$$
\bar{\sigma}_{c r}=f\left(\bar{\sigma}_{E}\right)
$$

The formulas of classical stability theory used to calculate the elasto-plastic critical stress are as follows:

The line formula [20]:

$$
\sigma_{c r}=\mathrm{A}-\frac{B}{1 /\left(\sqrt{\pi^{2} E / \sigma_{E}^{2}}\right)}
$$

The parabola formula [21]:

$$
\sigma_{c r}=\mathrm{A}-B \frac{\pi^{2} E}{\sigma_{E}}
$$

The Johnson-Ostenfeld formula [22] on plasticity correction:

$$
\sigma_{c r}=\sigma_{S}\left(1-\frac{\sigma_{S}}{4 \sigma_{E}}\right) ; \quad \sigma_{E}>\frac{\sigma_{S}}{2}
$$

Reconsider the format of Eq.(27)-(29) from which a formula in a more general form can be proposed as follows:

$$
\bar{\sigma}_{c r}=\left[A-B\left(\frac{1}{\bar{\sigma}_{E}}\right)^{c}\right]^{m}
$$

Based on the above equations, a five-parameter formula can be used to describe the stress of limit equilibrium state:

$$
\begin{aligned}
& \bar{\sigma}_{c r}=\left(A-B(n \phi)^{c}\right)^{m} \\
& \phi=\frac{\sigma_{0}}{\sigma_{E}}
\end{aligned}
$$

where $\phi$ and $n$ represent the state parameter of the ideal material and the synthesis factor, respectively. $\phi$ corresponds to the Euler stress, which is the linear failure stress, and $n$ represents all types of influential factors, such as initial deflection and residual stress. If a structure is perfect, the factor of material nonlinearity is only considered with the value of $n$ taken as 1 .

\section{Formulas for beams on elastic foundations: a spherical shell}

According to the methodology and procedures mentioned above, it is possible to solve the elastic-plastic stability of structures under pressure, including bars, stiffened plates, and panels.

4.1 The limit state of a beam on an elastic foundation

The bending equation for a beam on an elastic foundation, as shown in Fig.3, can be derived as follows [23]:

$$
D \frac{\mathrm{d}^{4} w}{\mathrm{~d} x^{4}}+T \frac{\mathrm{d}^{2} w}{\mathrm{~d} x^{2}}+k w=q .
$$




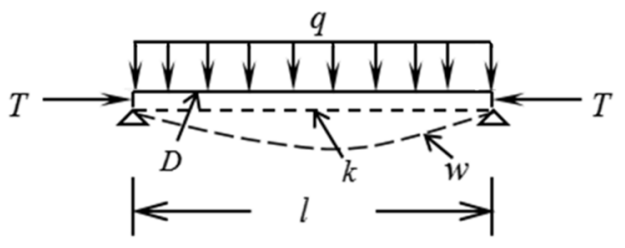

Fig. 3 A typical beam on an elastic foundation under uniform and axial loads

\subsection{The stable equilibrium equation of a spherical shell under uniform pressure}

Spherical and cylindrical shells, such as deep sea vehicles and submarines, are common structures in ocean engineering. Bending deformation of a stiffened cylindrical shell under hydraulic pressure can be replaced by a strip shell which is shown in Fig.4. The radial component of stresses $\sigma_{y}$ on both sides of the strip can produce a combined load $q_{y}$ in the opposite direction of the deflection $w$. Therefore, the loads $q_{y}$ can be added to Eq. (33) and the equilibrium equation takes the following form:

$$
D \frac{\mathrm{d}^{4} w}{\mathrm{~d} x^{4}}+T \frac{\mathrm{d}^{2} w}{\mathrm{~d} x^{2}}+k w=q+q_{y}
$$

(a)

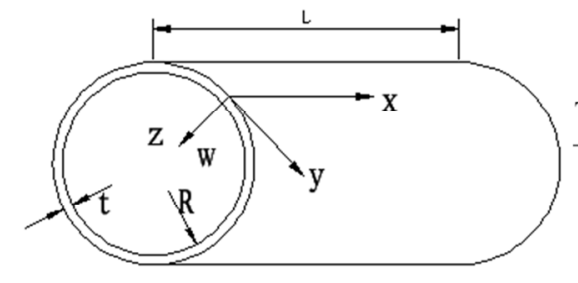

(b)

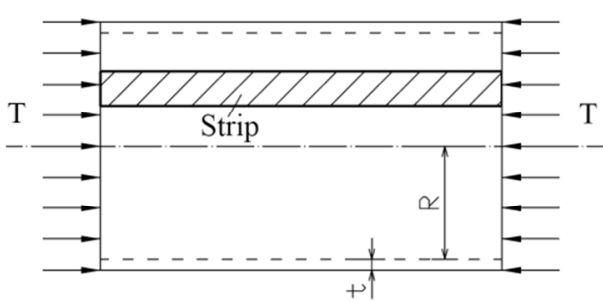

(c)

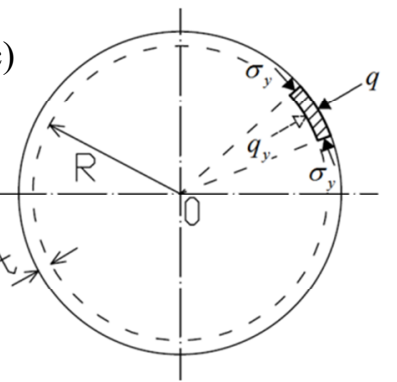

Fig. 4 Loads on a cylindrical shell: (a) Three-dimensional view (b) Elevation view (c) Sectional view

If a spherical shell buckles under uniform pressure, the buckling area is a spherical crown with a circular boundary as shown in Fig.5. The buckling area is symmetrical about the axis of the sphere $[24,25]$, as shown in Fig.5. Assuming a symmetric failure, we can simplify the equilibrium equation of a spherical shell to the form of the equilibrium equation of a beam on an elastic foundation.
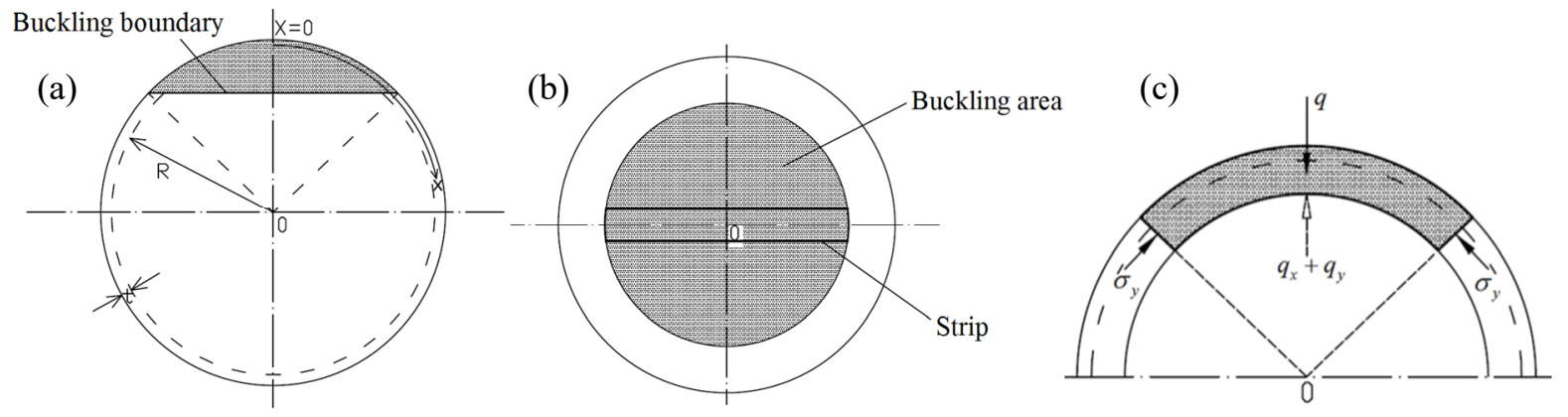

Fig. 5 Buckling area of a spherical shell: (a) Elevation view (b) Plan view (c) Force body diagram of a strip

Compared with a cylindrical shell, the strip of a spherical crown is a ring. Therefore, the strip of the spherical shell can be considered as the strip of the cylindrical shell with initial deflection. Similar to the cylinder, as shown in Fig. 4(c), the radial component of $\sigma_{y}$ can produce $q_{\mathrm{y}}$ and $\sigma_{x}$ can produce $q_{\mathrm{x}}$, as shown in Fig. 5(c). 
If a beam has an initial deflection $w_{0}$, the equation of equilibrium similar to that for beams on elastic foundations can be written as [26]:

$$
D \frac{\mathrm{d}^{4} w}{\mathrm{~d} x^{4}}+T \frac{\mathrm{d}^{2} w}{\mathrm{~d} x^{2}}=\left(q-q_{x}-q_{y}\right)-T \frac{\mathrm{d}^{2} w_{0}}{\mathrm{~d} x^{2}}
$$

The ring area taken from the spherical shell can be simplified to a beam with a small deflection. The curvature of the initial deflection of the beam is $1 / R$.

From the above derivations, it is shown that the tangent modulus theory and the double modulus theory can be applied to a cylindrical shell and a spherical shell to calculate their elasto-plastic buckling stress.

\section{Case Study}

In this paper, a spherical shell is selected to show how to calculate its critical stress.

5.1 Nonlinear characteristics of the material

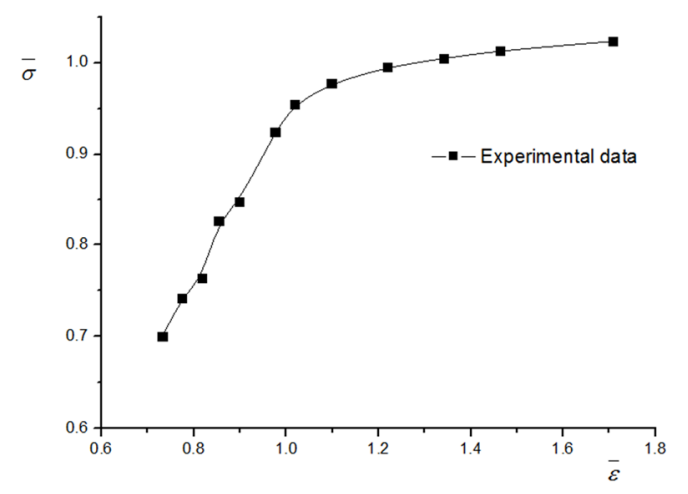

Fig. 6 Stress-strain $(\bar{\sigma} \sim \bar{\varepsilon})$ curve of the TC4 material

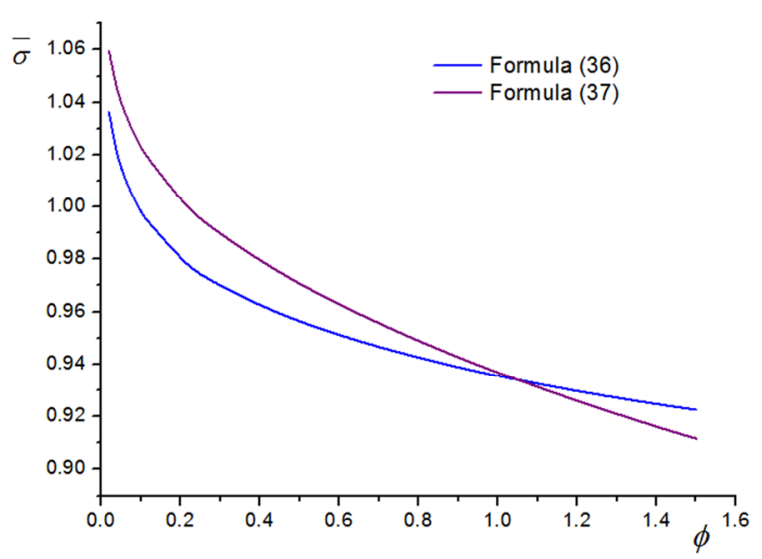

Fig. 7 Two types of modulus curves $(\bar{\sigma} \sim \phi)$

From the stress-strain curve $(\bar{\sigma} \sim \bar{\varepsilon})$ shown in Fig.6 and the modulus curve $(\bar{\sigma} \sim \phi)$ shown in Fig.7, one can determine the four material parameters $A, B, c$, and $m$, which are always calculated using the least-mean-square method and the experimental data.

The formula based on the tangent modulus theory can be derived as follows:

$$
\bar{\sigma}_{c r}=\left(0.350+1.4 \phi^{0.323}\right)^{-0.121}
$$

In a similar way, the formula based on the double modulus theory can be expressed as:

$$
\bar{\sigma}_{c r}=\left(3.05-2.39 \phi^{0.1}\right)^{0.16}
$$

The results from Eq. (36) and (37) are in very good agreement with the data from both modulus theories.

5.2 Formula for the critical buckling stress $\left(\bar{\sigma}_{c r}\right)$ and the ratio of yield stress to the Euler stress $(\phi)$

The expression for the critical buckling stress $\bar{\sigma}_{c r}$ can be derived from Eq. (31):

$$
\bar{\sigma}_{c r}=\left(\mathrm{A}-\mathrm{B} \phi^{c}\right)^{m}
$$


The Euler stress for calculating the stability of a spherical structure can be obtained as follows $[27,28]$ :

$$
\begin{aligned}
& \sigma_{E}=p_{E} R / 2 t \\
& p_{E}=\frac{2 E}{\sqrt{3\left(1-\mu^{2}\right)}}\left(\frac{t}{R}\right)^{2}
\end{aligned}
$$

Usually the factor $n$ corresponds to the initial shape and the failure feature of the structure. In this example, $n$ can be taken as 1 by assuming that the spherical shell is in a perfect state. By applying the classical expressions for elastic buckling from Eq. (31) and (39) to Eq. (38), the inelastic buckling stress formula can be established as follows:

$$
\bar{\sigma}_{c r}=\left(A-B\left(\frac{2 \sigma_{0} t}{p_{E} R}\right)^{c}\right)^{m}
$$

5.3 Comparison between the results calculated from both modulus theories and experimental data

Since the failure of the spherical structure is within the range of inelastic buckling, the inelastic buckling stress and the corresponding buckling pressures can be easily estimated from Eq. (38) and (39).

In order to verify the accuracy of the explicit expression presented in this paper, experimental results of models no. 1 and 2 from Pan et al. [29] and the MT-1 model from Yokota and Murata [30] are compared. The parameters mentioned in this paper are summarized in Table 1.

Table 1 Parameters of the tested models made of TC4

\begin{tabular}{|c|c|c|c|c|c|c|c|}
\hline \multicolumn{2}{|r|}{ Tested model } & $n$ & $P_{E X P}$ & $P_{c r}(t)$ & $P(t) / P_{c}$ & $P_{c r}(d)$ & $P(d) / P$ \\
\hline \multirow[t]{2}{*}{1} & $\begin{array}{l}\text { Average } t \text { of the } \\
\text { Northern Hemisphere }\end{array}$ & 1 & \multirow[t]{2}{*}{56} & 59.5 & 1.06 & 60.6 & 1.08 \\
\hline & $\begin{array}{l}\text { Average } t \text { of the } \\
\text { Southern Hemisphere }\end{array}$ & 1 & & 60.4 & 1.07 & 61.4 & 1.10 \\
\hline \multirow[t]{2}{*}{2} & $\begin{array}{l}\text { Average } t \text { of the } \\
\text { Northern Hemisphere }\end{array}$ & $1 / 8.2$ & \multirow[t]{2}{*}{58.29} & $\begin{array}{c}68.48 / \\
64.13\end{array}$ & $1.17 / 1.10$ & $\begin{array}{l}69.79 / \\
61.23\end{array}$ & $1.19 / 1.05$ \\
\hline & $\begin{array}{l}\text { Average } t \text { of the } \\
\text { Southern Hemisphere }\end{array}$ & $1 / 8.2$ & & $\begin{array}{c}68.42 / \\
64.07 \\
\end{array}$ & $1.17 / 1.10$ & $\begin{array}{r}69.72 / \\
61.16 \\
\end{array}$ & $1.19 / 1.05$ \\
\hline \multicolumn{2}{|r|}{ MT-1 } & 1 & 123 & 124.1 & 1.01 & 126.9 & 1.03 \\
\hline
\end{tabular}

\begin{tabular}{|c|c|c|c|}
\hline Tested model & 1 & 2 & MT-1 \\
\hline Inner radius $(\mathrm{mm})$ & 250 & 250 & 234 \\
\hline$t$ of the Northern Hemisphere $(\mathrm{mm})$ & $8.23-8.68$ & $9.42-9.71$ & \\
\hline Average $t$ of the Northern Hemisphere $(\mathrm{mm})$ & 8.3671 & 9.5916 & 16.00 \\
\hline$t$ of the Southern Hemisphere $(\mathrm{mm})$ & $7.96-8.73$ & $9.41-9.79$ & \\
\hline Average $t$ of the Southern Hemisphere $(\mathrm{mm})$ & 8.4849 & 9.5824 & 16.00 \\
\hline Test collapse pressure $(\mathrm{MPa})$ & 56.00 & 58.29 & 123.00 \\
\hline
\end{tabular}

Table 2 Comparison between the calculated values and experimental results [29] for a spherical shell 
Table 2 shows that the results from the tangent modulus theory are more consistent with the experimental data. In the inelastic column theory [6], the critical loads should be between the results from the tangent modulus and double modulus theories. The tangent theory result is considered as the lower limit and the double modulus theory result is considered as the upper limit. For further verification, these results will be compared with the Finite Element method (FEM) results from [31] shown in Fig.8. As shown by Fig.8, the relative discrepancy between both modulus theories and FEM is about 7\%. This means that both modulus theories can produce reasonable results for thin-walled structures. However, such discrepancy may become greater with an increase in the value of $t / R$ and it may amount to more than $11 \%$. This may also indicate the limit of both modulus theories. In other words, both theories may be inappropriate for thick structures.

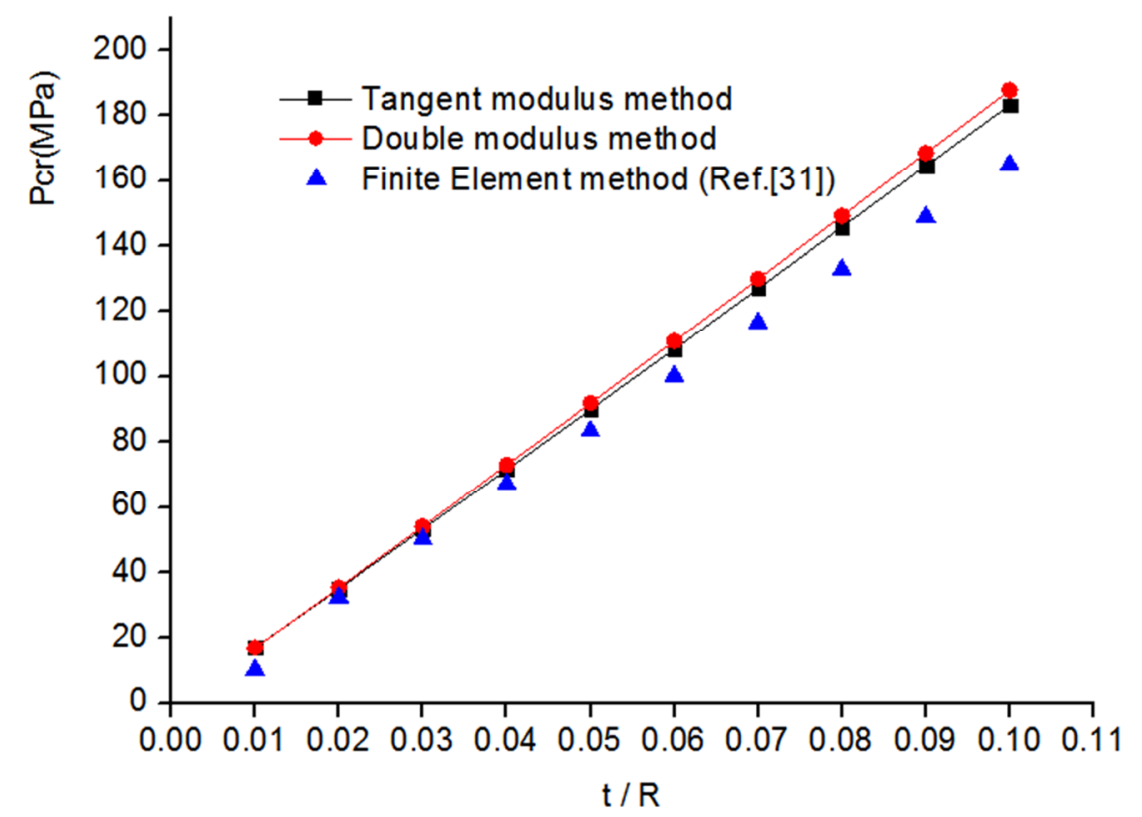

Fig. 8 Critical loads from the current methods and the finite element method [31]

5.4 Comparison between the experimental and calculated results for a spherical shell made of 304 stainless steel

Firstly, the stress-strain curve of 304 stainless steel [31] can be expressed as

$$
\bar{\sigma}=\sqrt[4.123]{0.1(\bar{\varepsilon}-1)}
$$

Secondly, expressions relating to the two modulus theories can be derived.

Based on the tangent modulus theory, the expression is as follows:

$$
\bar{\sigma}=\left(-1.0 \times 10^{-8}+4.64 \phi^{0.41}\right)^{-0.59}
$$

Based on the double modulus theory, it is

$$
\bar{\sigma}=\left(-0.127+0.477 \phi^{-0.328}\right)^{7.31}
$$

The final calculation results and the comparison with the experiment are shown in Table 3. 
Table 3 Comparison of ultimate strength obtained from the experiment [31]

\begin{tabular}{|c|c|c|c|c|c|c|c|c|c|c|}
\hline Index. & $\begin{array}{c}R \max \\
(\mathrm{mm})\end{array}$ & $\begin{array}{c}t \max \\
(\mathrm{mm})\end{array}$ & $\phi$ & $n$ & $\bar{\sigma}_{c r}$ & $\begin{array}{c}P_{c r}(\mathrm{t}) \\
(\mathrm{MPa})\end{array}$ & $\begin{array}{c}P_{c r}(\mathrm{~d}) \\
(\mathrm{MPa})\end{array}$ & $\begin{array}{c}P_{E X P} \\
(\mathrm{MPa})\end{array}$ & $P_{c r}(\mathrm{t}) / P_{E X P}$ & $P_{c r}(\mathrm{~d}) / P_{E X P}$ \\
\hline 1 & 74.485 & 0.422 & 0.334 & 1 & 0.529 & 1.665 & 2.049 & 1.708 & 0.97 & 1.19 \\
\hline 2 & 74.517 & 0.432 & 0.307 & 1 & 0.540 & 1.770 & 2.186 & 1.956 & 0.90 & 1.12 \\
\hline 3 & 74.285 & 0.426 & 0.317 & 1 & 0.536 & 1.734 & 2.139 & 1.773 & 0.98 & 1.21 \\
\hline 4 & 74.754 & 0.401 & 0.366 & 1 & 0.518 & 1.487 & 1.821 & 1.330 & 1.11 & 1.37 \\
\hline 5 & 74.501 & 0.414 & 0.334 & 1 & 0.529 & 1.665 & 2.049 & 1.594 & 1.04 & 1.29 \\
\hline
\end{tabular}

From Table 3, it can be concluded that the results calculated by the expression from the tangent modulus theory are more accurate than those from the double modulus theory. The results are consistent with the findings from Shanley's theory [6]. According to this theory, the critical load is between the values from the tangent modulus theory (the lower limit) and from the double modulus theory (the upper limit/ asymptotic limit).However, in reality, the buckling load value may be closer to that from the tangent modulus theory because some defects should be considered in calculations, e.g. manufacturing defects and geometric inaccuracies. Compared to the result of FEA, the tangent modulus theory result differs by $10 \%$ at the maximum. However, in the case of the double modulus theory result, the maximum difference is above $30 \%$. This indicates that an optimized $n$ may be needed to get more accurate results for the double modulus theory.

\section{Conclusions}

In this paper, an explicit expression for correlating the critical stress and the Euler stress has been proposed. The synthesis factor $n$ introduced in the newly-developed formula is used to reflect imperfection. This formula is further applied to the calculation of buckling loads of stiffened plates in a spherical shell, based on the tangent modulus and the double modulus theory. It is found that there is a $10 \%$ difference between the results from the tangent modulus theory and the experimental results. In addition, it is found that the double modulus theory may result in a bigger discrepancy than the tangent modulus theory. The maximum discrepancy is more than $20 \%$.

Overall, both the tangent modulus theory and the double modulus theory can be used to calculate the ultimate strength of a cylindrical shell or a spherical shell by means of this explicit formula. Different materials correspond to different parameters $\mathrm{A}, \mathrm{B}, c$, and $m$. It is evident that the inclusion of these parameters in this explicit formula can improve the accuracy when calculating the critical stress.

\section{Nomenclature}

\begin{tabular}{|c|l|}
\hline$\sigma_{c r}$ & critical buckling stress \\
\hline$\sigma_{E}$ & elastic buckling stress(Euler stress) \\
\hline$E_{t}$ & tangent modulus \\
\hline$E$ & elastic modulus \\
\hline$\sigma / \varepsilon$ & stress/strain of actual material \\
\hline$E_{r}$ & double modulus \\
\hline
\end{tabular}




\begin{tabular}{|c|c|}
\hline$\sigma_{0}$ & yield stress of material or its substitute \\
\hline$\sigma_{L} / \varepsilon_{L}$ & stress/strain of perfect material \\
\hline W & strain energy density \\
\hline$W_{\sigma}^{\prime}$ & derivative of W with respect to $\sigma$ \\
\hline$\varepsilon_{t}$ & tangent strain \\
\hline$\varepsilon_{0}$ & yield strain of a material or its substitute \\
\hline$\varepsilon_{c r}$ & strain of critical buckling \\
\hline$l$ & length of a compression bar \\
\hline$r$ & radius of inertia \\
\hline$D$ & bending stiffness \\
\hline$w$ & deflection \\
\hline$T$ & axial pressure of a bar \\
\hline$q$ & laterally distributed load \\
\hline$k$ & elastic stiffness \\
\hline$p_{\mathrm{E}}$ & collapse pressure due to elastic buckling \\
\hline$\mu$ & Poisson's ratio \\
\hline$t$ & shell thickness \\
\hline$R$ & mean radius of a spherical shell \\
\hline$\phi$ & ratio of yield stress to the Euler stress \\
\hline$n$ & synthesis factor \\
\hline$P_{c r}(t)$ & ultimate strength of spherical shells with the tangent modulus \\
\hline$P_{c r}(d)$ & ultimate strength of spherical shells with the double modulus \\
\hline$P_{E X P}$ & experimental results for ultimate strength of spherical shells \\
\hline
\end{tabular}

\section{Acknowledgments}

This study has been supported by two grants. The first is provided by the National Science Foundation of China (Grant No.51609134) and the second by the State Key Laboratory of Ocean Engineering of Shanghai Jiao Tong University (Grant No.1610).

\section{REFERENCES}

[1] Euler, L. Sur La force de conlonnes. Memoires del's Academie de Berlin 1759.

[2] Engesser,F. Ueber die Knickfestigkeit gerader Stabe, Zeitschrift fur Architektur and Ingenieurwesen 1889,35 .

[3] Engesser,F. “Knickfragen”. Schweitzerische Bauzeitung 1895, 26.

[4] Von Kármán, T. Untensuchungen üder Knickfestigkeit, Mitteilungen uder Forschungsarbeiten auf dem Gebiete des Ingenieurwesens, Veiein Deutscher Ingenieure, Berlin Heft 81, 1910.

[5] Shanley, F.R. The Column Paradox, Journal of Aeronautical Sciences 1946, 13(12), 678-678. https://doi.org/10.2514/8.11478 
[6] Shanley, F.R. Inelastic Column Theory, Journal of Aeronautical Sciences 1947, 14(5), 261-268. https://doi.org/10.2514/8.1346

[7] Chen, C.N. A finite element study on Shanley's plastic buckling theory of short columns, Computers \& Structures 1996, 59(5), 975-982. https://doi.org/10.1016/0045-7949(95)00281-2

[8] Becque, J. The application of plastic flow theory to inelastic column buckling, International Journal of Mechanical Sciences 2016,111-112(6), 116-124.https://doi.org/10.1016/j.ijmecsci.2016.04.005

[9] Duberg, J.E. and Wilder T.W., III, Column behavior in the plastic stress range, Journal of the Aeronautical Sciences 1950. 17(6), 323-327. https://doi.org/10.2514/8.1642

[10] Hutchinson, J. W. On the postbuckling behavior of imperfection-sensitive structures in the plastic range, Journal Applied Mechanics 1972, 39(1), 155-162. https://doi.org/10.1115/1.3422605

[11] Reynolds, T.E. Inelastic Lobar Buckling of Cylindrical Shells under External Hydrostatic Pressure, David Taylor Model Basin Report 1392, 1960.

[12] Krenzke, M.A., Kiernan T.J. Test of stiffened and unstiffened machined spherical shells under external hydrostatic pressure, David Taylor Model Basin, report 1741, S-R0110101, 1963.

[13] Schneider, S.; Schneider, S.G.; Marques da Silva, H.; Neto,C.M. Study of the non-linear stress-strain behavior in Ti-Nb-Zr alloys, Materials Research 2005, 8(4),435-438.

https://doi.org/10.1590/S1516-14392005000400013

[14] Pranesh, S.B., Kumar, D., Subramanian, V.A., Sathianarayanan, D. Non-linear buckling analysis of imperfect thin spherical pressure shell for manned submersible, Journal of Ocean Engineering and Science 2017, 2(4), 293-300. https://doi.org/10.1016/j.joes.2017.11.001

[15] Luo, P.L., Luo, H.W., Tong, F.S. The Influence of Pre buckling Deformations and Stresses on the Buckling of the Spherical Shell,International Journal of Offshore and Polar Engineering 1991,1(4).

[16] Luo, P.L.The establishment and development of a combined theory of strength and stability based on innovative Hooke's Law,Journal of Harbin Engineering University 2008, 29(7), 641-650. In Chinese

[17] Ramberg, W., Osgood, W.R. Description of stress-strain curves by three parameters, National Advisory Committee for Aeronautics 1943, 7, 1-32.

[18] Kim, J.R.R., Rondal, J. Strength curves for aluminium alloy columns. Engineering Structures 2000, 22(11), 1505-1517. https://doi.org/10.1016/S0141-0296(99)00089-9

[19] Gerard, G.; Becker, H. Handbook of Structure Stability Part I : Buckling of Flat Plates, NACATN7381.New York University N.Y. 1957.

[20] Rodal, J. and Maquoi, R.Single. Single equation for SSRC column strength curves. Journal of Structural Division, ASCE 1979, 105(1):247-250.

[21] Bleich, F. Buckling Strength of Metal Structures, New York: McGraw-Hill Book Company 1952.

[22] Paik, J.K., Thayamballi, A.K., Wang, G., Kim, B.J. On advanced buckling and ultimate strength design of ship plating, annual meeting preprints of the society of naval architects and marine engineers $\mathbf{2 0 0 0}, 1$, 13-34.

[23] Essa, S. Analysis of elastic beams on linear and nonlinear foundations using finite difference method, 4th International Engineering Conference on Developments in Civil \& Computer Engineering Applications 2018, 236-250. https://doi.org/10.23918/iec2018.18

[24] Koga, T.; Hoff, N.J. The axisymmetric buckling of initially imperfect complete spherical shells, International Journal of Solids and Structures 1969, 5(7), 679-697. https://doi.org/10.1016/0020-7683(69)90088-2

[25] Zheng, Y.S., Liu, Z.F., Zhang, D.W. Collapse pressure of spherical shells with initial imperfections under uniform pressure, Shipbuilding of China 1986, 1, 49-58.

[26] H'y ca, M. Optimization of Initial Deflection of a Beam-Column (a Planar Problem). Proceedings in Applied Mathematics and Mechanics 2006, 6, 223-224. https://doi.org/10.1002/pamm.200610092

[27] Pan, B.B., Cui, W.C. An overview of buckling and ultimate strength of spherical pressure hull under external pressure, Marine Structures 2010, 23(3), 227-240.

https://doi.org/10.1016/j.marstruc.2010.07.005

[28] Zoelly, R. Über ein Knickungs problem an der Kugelschale. Thesis, Zürich 1915. 
[29] Pan, B.B., Cui, W.C., Shen, Y.S. Experimental verification of the new ultimate strength equation of spherical pressure shells. Marine Structure 2012, 29(1), 169-176. https://doi.org/10.1016/j.marstruc.2012.05.007

[30] Yokota, K.; Murata, T. Research on pressure shell for deep submergence research vehicles made of titanium alloy,Technical Review, Mitsubishi Heavy Industries, Ltd. 1986, 23(4), 440-442.

[31] Zhang, J., Zhang, M., Tang, W.X., Wang, W., Wang, M.L.. Buckling of spherical shells subjected to external pressure: A comparison of experimental and theoretical data, Thin-Walled Structures 2017, 111(2), 58-64. https://doi.org/10.1016/j.tws.2016.11.012

Submitted: $\quad 05.01 .2018$

Accepted: $\quad 12.10 .2018$
Zhixin Xiong

College of Ocean Science and

Engineering, Shanghai Maritime

University, Shanghai 201306, China

Peilin Luo

College of Shipbuilding Engineering,

Harbin Engineering University, Harbin,

Heilongjiang 150001, China

Xiaochuan $\mathrm{Yu}$

(Corresponding Author: xyu5@uno.edu)

School of Naval Architecture and Marine

Engineering, University of New Orleans,

New Orleans, LA 70148, USA 